\title{
Changing our thinking about changing their thinking in older adulthood
}

\section{Can we really teach older adults to change their thinking?}

It is well-established that as people age, deterioration in cognitive abilities including processing speed, memory, and cognitive flexibility occurs, although vast individual differences occur in the rate and consequences of this decline (Christensen, 2001). Anxiety and depression in late life are also associated with specific cognitive deficits in memory and executive functioning that may impact on new learning (Yochim et al., 2013). Therefore, it is possible that cognitive changes make it more difficult for older adults to learn how to change their thinking particularly in the context of psychological therapy.

The most well-established psychological intervention for reducing anxiety and depression, and emotional distress across a range of psychiatric disorders, is cognitive behavioral therapy or CBT (Hofmann et al., 2012). An important component of CBT is cognitive therapy which teaches individuals to identify and change irrational thinking. The process of identifying and challenging irrational thinking requires the ability to learn a new skill, identify irrational thoughts, and be flexible in thinking in order to generate plausible and relevant evidence to refute the irrational thoughts and generate a more helpful thought. The complexity of cognitive restructuring, coupled with the natural declines in cognitive ability, and cognitive difficulties associated with anxiety and depression in older adults, therefore could present a therapeutic challenge for clinicians and clients. In fact, it has been claimed that older adults are unable to engage in the abstract thinking and complex cognitive skills required in cognitive therapy (Church, 1983), and is a common view held among therapists. Heterogeneity is the norm in older adult populations with vast differences in the way that individuals' age. So it is simplistic to suggest that age-related changes will impact on treatment outcomes and therapy delivery in all older adults. However, given that cognitive changes are likely to occur across the older adult age range, we need to establish the impact of these cognitive changes on cognitive therapy outcomes. Is it true that older adults are less able to identify and challenge their thinking?

\section{Evidence for cognitive behavioral therapy in older adults}

Meta-analyses and systematic reviews of randomized controlled trials for treating anxiety and depression in older adults have found that CBT is efficacious (Gould et al., 2012a; Gould et al., $2012 b)$. There are, however, vast differences in the effect sizes found in individuals studies, and this is likely to relate to differences in the methodology used, the populations studied and possibly to different emphasis on the specific CBT elements taught. The manualized programs used in most studies were developed in younger adults and adapted to older adult populations (e.g. Coping with Depression Course (Lewinson et al., 1984), and Mastery of your Anxiety and Worry course (Craske et al., 1992)); however, some have been developed specifically for older adults (e.g. Ageing Wisely (Wuthrich, 2009)). These programs contain similar therapy elements including cognitive restructuring as a core skill. Compartmentalizing studies have not been adequately conducted in older populations, particularly in the anxiety disorders, and so it is not clear whether declines in cognitive ability impact specifically on the ability of older adults to learn and benefit from cognitive restructuring.

\section{Impact of cognitive ability on CBT effectiveness}

Some very promising research recently examined the impact on executive functioning and cognitive flexibility on both overall CBT treatment response and the ability to specifically learn how to challenge irrational thinking in two different populations. In a series of experiments in older adults with co-occurring mood and anxiety disorders (Johnco et al., 2013; 2014; 2015), it was found that when cognitive restructuring was taught in a single 1-hour session, older adults with reduced cognitive flexibility in both clinical and nonclinical populations had more difficulty learning the 
cognitive restructuring techniques than those with better cognitive flexibility, while older adults with normal cognitive flexibility were able to successful learn to use cognitive reappraisal to challenge their thinking (Johnco et al., 2013; 2015). Perhaps this is not surprising, however, at the end of the CBT program in which participants were able to practice cognitive restructuring over 12 weeks, cognitive flexibility was not found to significantly impact on the ability to learn cognitive therapy skills (Johnco et al., 2014). This suggests that with repeated practice during the course of group CBT, even participants with reduced cognitive flexibility can learn to identify and challenge irrational thinking successfully. This was similar to the findings examining the impact of executive functioning on CBT efficacy in older adults with Generalized Anxiety Disorder (Mohlman, 2013), who also found that executive functioning was not significantly related to overall treatment response for CBT. These studies together suggest that even for older adults with reduced executive functioning and cognitive flexibility skills who might have initial difficulty learning cognitive disputing skills, with repeated practice during group CBT, are capable of learning to identify and challenge irrational thinking, and that executive functioning and cognitive flexibility at pre-treatment do not impact significantly on overall treatment outcome. While these results are promising, more research examining the impact of normal cognitive changes on cognitive therapy skill development and delivery is clearly needed.

\section{Positive reappraisal}

Conversely, there is emerging research that there might be particular features of aging that make changing negative thinking in some populations easier than in younger populations. Positive reappraisal is a cognitive reappraisal strategy of identifying positive meaning within a stressful experience that has been shown to be used more frequently and effectively in older adults than younger adults (Nowlan et al., 2015a). This increased ability to identify the "silver lining" in stressful events is an interesting finding that may have potential utility in therapy contexts. The reasons for this increased use of positive reappraisal to cope with stressors with increasing age is unknown, however, it is likely to be related to increased life experience and wisdom. It is also explained by theoretical models (Socioemotive Selectivity Theory: Carstensen et al., 1999; and Strength and Vulnerability Integration model: Charles, 2010) and experimental findings replicated across a range of paradigms showing that older adults are more inclined than younger adults to attend to positive stimuli, seek out and maintain positive mood states, and avoid negative mood states (Carstensen et al., 2000; Mather, 2012). Potentially, this tendency to attend to positive information and avoid negative information, can be utilized to teach older adults to change their thinking about negative emotions or situations. In a novel series of studies, it was recently shown that older adults can be taught to change their thinking to use positive reappraisal to look for the positives in negative situations, and further was found to relate to improvements in wellbeing and coping in samples of older adults with chronic illness (i.e. diabetes) and chronic stressors (Nowlan et al., 2015b; Nowlan et al., 2016). Hence, interventions in older adults that harness this increased attention to positive outcomes may be of great promise.

\section{Dementia}

It is important that the normal age-related cognitive changes referred to in this paper are differentiated from non-normal cognitive decline such as in mild cognitive impairment (MCI) or dementia. Individuals with MCI or dementia are very likely to struggle to apply cognitive interventions in conventional ways, and hence most psychological therapy approaches with these populations are predominantly behavioral in nature. However, there is emerging evidence that even in older adults with dementia that reminiscence therapy holds great promise for improving psychological wellbeing (Bhar, 2014). Reminiscence therapy relies on identifying personal strengths and previous challenges overcome by individuals by activating long-term memories which are less susceptible to the dementing process, at least in the early stages, and therefore can be utilized to increase individuals' abilities to cope with current situations.

\section{Conclusion}

So, what does the evidence tell us about the ability of older adults to change their thinking? The evidence clearly indicates that most older adults can utilize cognitive interventions in helpful ways. In the case of cognitive therapy, the majority of older adults are capable of learning and benefiting from cognitive restructuring skills despite any normal age-related changes that occur. Further, for those older adults who have some difficulties with executive functioning and/or cognitive flexibility, these deficits appear not to lead to significant reductions in therapeutic efficacy 
of cognitive restructuring or CBT overall with repeated practice. There is also promising evidence that cognitive interventions to increase the use of positive reappraisal as a coping strategy might be particularly useful for older adults with chronic stressors or illnesses. Finally, even in older adults with dementia, promising research on reminiscence therapy indicates that cognitive strategies can still have a significant impact on improving emotional well-being. So, as clinicians, we need to be careful to check our thinking about older adults' ability to benefit from changing their thinking.

\section{VIVIANA M. WUTHRICH}

Department of Psychology, Centre for Emotional Health, Macquarie University, Sydney, Australia Email: viviana.wuthrich@mq.edu.au

\section{References}

Bhar, S. (2014). Remiscence therapy: a review. In N. A. Pachana and K. Laidlaw (eds.), Oxford Handbook of Clinical Geropsychology (pp. 675-690). Oxford: Oxford University Press.

Carstensen, L. L., Isaacowitz, D. M. and Charles, S. T. (1999). Taking time seriously: a theory of socioemotional selectivity. American Psychologist, 54, 165-181. doi:10.1037/0003-066X.54.3.165.

Carstensen, L. L., Pasupathi, M., Mayr, U. and Nesselroade, J. R. (2000). Emotional experience in everyday life across the adult life span. Fournal of Personality E Social Psychology, 79, 644-655. doi:10.1037/0022-3514.79.4.644.

Charles, S. T. (2010). Strength and vulnerability integration: a model of emotional well-being across adulthood. Psychological Bulletin, 136, 1068-1091. doi:10.1037/a0021232.

Christensen, H. (2001). What cognitive changes can be expected with normal aging? Australian and New Zealand Fournal of Psychiatry, 35, 768-775. doi:10.1046/j.1440-1614.2001.00966.x.

Church, M. (1983). Psychological therapy with elderly people. Bulletin of the British Psychological Society, 36, 110-112.

Craske, M. G., Barlow, D. H. and O'Leary, T. (1992). Mastery of Your Anxiety and Worry. Albany, New York: Psychological Corporation.

Gould, R. L., Coulson, M. C. and Howard, R. J. (2012b). Efficacy of cognitive behavioral therapy for anxiety disorders in older people: a meta-analysis and meta-regression of randomized controlled trials. Fournal of the American Geriatrics Society, 60, 218-229.
Gould, R. L., Coulson, M.C. and Howard, R. J. (2012a). Cognitive behavioral therapy for depression in older people: a meta-analysis and meta-regression of randomized controlled trials. Fournal of the American Geriatrics Society, 60, 1817-1830.

Hofmann, S. G., Asnaani, A., Vonk, I. J. J., Sawyer, A. T. and Fang, A. (2012). The efficacy of cognitive behavioral therapy: a review of meta-analyses. Cognitive Therapy and Research, 36, 427-440.

Johnco, C., Wuthrich, V. M. and Rapee, R. M. (2013). The role of cognitive flexibility in cognitive restructuring skill acquisition among older adults. Fournal of Anxiety Disorders, 27, 576-584. doi:10.1016/j.janxdis.2012.10.004.

Johnco, C., Wuthrich, V. M. and Rapee, R. M. (2014). The influence of cognitive flexibility on treatment outcome and cognitive restructuring skills acquisition during cognitive behavioural treatment for anxiety and depression in older adults: results of a pilot study. Behaviour Research and Therapy, 57, 55-64.

Johnco, C., Wuthrich, V. M. and Rapee, R. M. (2015). The impact of late-life anxiety and depression on cognitive flexibility and cognitive restructuring skill acquisition. Depression and Anxiety, 32, 754-762. doi: $10.1002 /$ da.22375.

Lewinsohn, P. M., Antonuccio, D. O., Breckenridge, J. S. and Teri, L. (1984). The 'Coping with Depression' Course. Eugene: Castalia Publishing Company.

Mather, M. (2012). The emotion paradox in the aging brain. Annals of the New York Academy of Sciences, 1251, 33-49. doi:10.1111/j.1749-6632.2012.06471.x.

Mohlman, J. (2013). Executive skills in older adults with GAD: relations with clinial variables and CBT outcome. Fournal of Anxiety Disorders, 27, 131-139.

Nowlan, J. S., Wuthrich, V. M. and Rapee, R. M. (2015a). Positive reappraisal in older adults: a systematic literature review. Aging $\mathcal{E}$ Mental Health, 19, 475-484. doi: https://doi.org/10.1080/13607863.2014.954528.

Nowlan, J. S., Wuthrich, V. M. and Rapee, R. M. (2015b). Increasing older adults' use of positive reappraisal coping for chronic physical illness. Clinical Gerontologist, 38, 428-437. doi:10.1080/07317115.2015.1067274.

Nowlan, J., Wuthrich, V. M. and Rapee, R. M. (2016). A comparison of single-session positive reappraisal, cognitive restructuring and supportive counselling for older adults with type 2 diabetes. Cognitive Therapy and Research, 40, 216-229. doi:10.1007/s10608-015-9737-x.

Wuthrich, V. M. (2009). Ageing Wisely: Group Program for the Treatment of Worry and Low Mood in Older Adults. Sydney: Centre for Emotional Health, Department of Psychology, Macquarie University.

Yochim, B. P., Mueller, A. E. and Segal, D. L. (2013). Late life anxiety is associated with decreased memory and executive functioning in community dwelling older adults. Fournal of Anxiety Disorders, 27, 567-575. Available at https://doi.org/10.1016/j.janxdis.2012.10.010. 